\title{
Information fusion in recognition of Saudi Arabia license plates
}

\author{
HAMDAN AL-HARBI, RAMI AL-HMOUZ \\ Department of Electrical and Computer Engineering \\ King Abdulaziz University \\ Jeddah \\ SAUDI ARABIA
}

\begin{abstract}
The license plate recognition (LPR) system in Saudi Arabia is a system used to identify vehicle license plates automatically. It is used in many places such as airports, highways, and parking lots. The efficiency of the system depends on the image quality, weather conditions, location of plates, and the variations of license plates. The license plates in the Kingdom of Saudi Arabia are different from other license plates in other countries because they are written in both Arabic and English languages. This could be exploited to integrate the recognition results from both languages in a way to increase the efficiency of the system and reduce the errors that could affect the recognition of license plates. Instead of one LPR system, we have two independent LPR systems, and the results of both systems could be fused to increase the system's ability of reading cars' plates.
\end{abstract}

Key-Words: - License plate recognition, information fusion, Saudi Arabia license plates

Received: April 12, 2020. Revised: October 22, 2020. Accepted: November 19, 2020. Published: December 8, 2020.

\section{Introduction}

License Plate Recognition (LPR) is an image technology used to identify plates for their vehicles. In areas such as surveillance and identification of vehicles, it is a must to have an automatic system that detects and recognizes license plates [1].

There are three common tasks considered to achieve the license plate recognition. These tasks are plate localization, character segmentation, and optical recognition. Locating a license plate inside a larger image is the most crucial and key step of automatic license plate recognition and has previously been accomplished in many ways. The contrast between the background (license plate) and the foreground (letters) is significant for the license plate localization. There are various methods for locating the license plate such as color edge detection [2], fuzzy systems [3], weight-based density maps [4], edge statistics [5] [6], Discrete Fourier transform pate signal [7], and morphological operations [8].

After the license plate image is located and extracted from a picture, then individual characters in the license plate are segmented. Character segmentation is the procedure of extracting the image characters from the license plate image [9].

License Plate Recognition is also known as Optical Character Recognition (OCR), and it is the last stage of the vehicle license plate recognition system. In this phase, the plate character images that have been taken out from the license plate image have to be identified and recognized. After segmenting the letters and numbers in the license plate, the characters are then fed to a classifier to recognize the character, examples of such classifiers include template matching [10], neural network [4], support vector machine [11], and Naïve Bayes [12].

A Fusion is the process of combining a group of units (or information) in such way they integrate with each other to form one unit. In this sense, fusion has been researched in the different levels of license plate systems. In [6], a probabilistic model was presented to combines the plate locations from different thresholds of car image. While in [12], a fusion process is applied to recognize the character image in a method called pixel fusion.

The Saudi Arabia License plates have a very significant characteristics which is that all areas in the plates have a dark rectangle around the 
numbers, letters, and symbols in. Another significant characteristic of the Saudi license plates is the position of numbers and letters i.e. numbers and letters do not mix with each other.

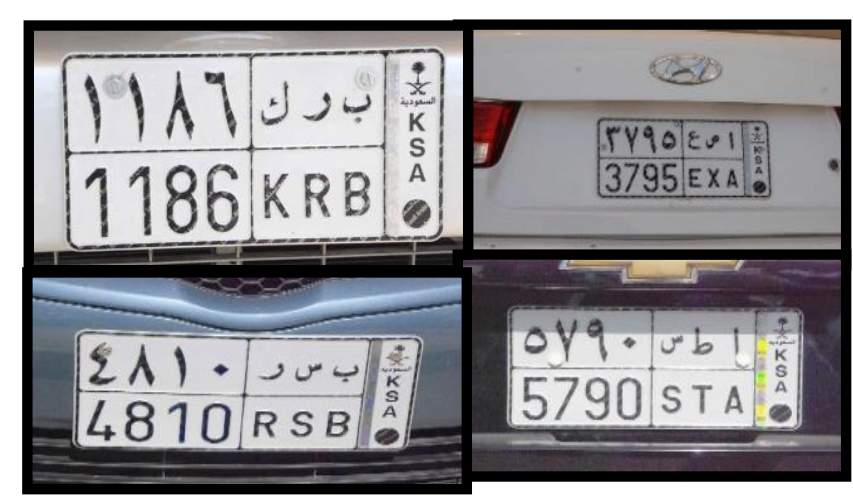

Fig.1. Saudi Arabia License plates samples.

On the top of all that, Saudi Arabia license plate consists of two corresponding letters (Arabic, English) that enable any LPR system to recognize the plates from the Arabic characters, English characters, or even both. (Figure1) shows samples of Saudi Arabia License plates.

The study by Sarfraz [13] was the first of its kind for Saudi Arabian license plates. The system was tested over many car images and was proved $95 \%$ accurate. Alginahi [14] proposed a system that recognizes both old and new Saudi license plates. The license plates are detected by searching the vertical lines, then the width to length ratio is used to locate the plate. The system provided a total character recognition rate of $98.63 \%$. Shaaban [15] proposed a system where a neural network classifier is used to recognize the characters based on edge moment invariants and principal component analysis features of wavelet coefficient matrix. The proposed system achieved a recognition rate of $97.6 \%$. Khalid Almustafa [16, 17] proposed an algorithm that uses pixel density to recognize characters in license plates. Maglad [18, 19] proposed a system that uses radial basis function (RBF) in neural networks $(\mathrm{NN})$ for the detection and recognition of plates. The system was able to detect the license plate of the Saudi cars. Deriche [20] proposed a hybrid system of RFID and license plate recognition. The ALPR module was used to identify unauthorized vehicles achieving a recognition accuracy of license plate numbers with $94 \%$.
The motivation of this paper is to develop a system that could detect and extract a license plate from a vehicle image and recognize both English and Arabic characters and numbers on Saudi license plates. The study will shed light on the reality of the fusion of Arabic and English characters for accurate license plate recognition.

The structure of the paper is as follows, next, we will describe the Saudi Arabia license plates. later, the steps of recognition the license plates are shown in in section 3. In Section 4, we show the fusion process in the recognition of Arabic and English texts of Saudi Arabia license plates. Results and discussions are placed in section 6. Finally, the paper will be concluded in section 6 .

\section{Saudi Arabia License Plate Description}

The license plates of Saudi cars consist of seven alphanumerical Arabic and English characters (refer to Figure 1). The first four characters in the Arabic and English texts are numeric $(\cdot, 1, \ldots, 9.0,1$, $\ldots, 9)$, and the remaining three characters are alphabetic letters.

The new Saudi license plates are white and contain Arabic letters and numbers, English letters, and Hindi numbers (refer to Figure 2). The letters are three characters, while the numbers are up to 4 characters. Region 3 (R 3) with the Hindi numbers is on the upper far left. Region 5 (R 5) with the Arabic numbers are on the lower left. Region 2 (R 2) The Arabic letters is on the upper middle part. Region 4 (R 4) with the English letters is on the lower middle part. Region 1 (R 1), a vertical colored region on the right side has the symbol of the kingdom as well as the letters KSA (abbreviating the name of the country, Kingdom of Saudi Arabia).

\begin{tabular}{|c|c|c|}
\hline Region 3 (R3) & Region 2 (R2) & 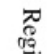 \\
\hline Region 5 (R5) & Region 4 (R4) & 刃्ల \\
\hline
\end{tabular}

Fig.2. Saudi Arabia License structure.

There are only 17 characters are used in Saudi Arabia license plates. The Arabic characters that أ، ب، ح، د، ر، س، ص، ط، ( ) 
(ع). While, the corresponding English characters are shown in Table 1.

Table1: Arabic and English characters of Saudi license plates.

\begin{tabular}{|c|c|c|c|}
\hline $\begin{array}{l}\text { Arabic } \\
\text { letters }\end{array}$ & $\begin{array}{c}\text { English } \\
\text { letters }\end{array}$ & $\begin{array}{l}\text { Arabic } \\
\text { letters }\end{array}$ & $\begin{array}{c}\text { English } \\
\text { letters }\end{array}$ \\
\hline i & A & ق & $\mathrm{G}$ \\
\hline ب & B & ك) & $\mathrm{K}$ \\
\hline$\tau$ & $\mathrm{J}$ & J & $\mathrm{L}$ \\
\hline 2 & $\mathrm{D}$ & p & Z \\
\hline ر & $\mathrm{R}$ & ن ن & $\mathrm{N}$ \\
\hline س & $\mathrm{S}$ & هـ & $\mathrm{H}$ \\
\hline ص & $\mathrm{X}$ & g & $\mathrm{U}$ \\
\hline$b$ & $\mathrm{~T}$ & $\checkmark$ & $\mathrm{V}$ \\
\hline$\varepsilon$ & $\mathrm{E}$ & & \\
\hline
\end{tabular}

It should be noted that, Arabic language consist of 28 characters, while the English language consist of 27 characters. Saudi license plates use only 17 corresponding of Arabic and English characters.

\section{License Plate Recognition}

Any LPR system consists of the following:

1- Image acquiring:

The general aim of image Acquisition is to transform an optical image (Real World Data) into an array of numerical data, which could be later manipulated by an LPR systems. In some applications (i.e., car park, speed limit), the system is triggered to start the recognition process and the camera is focused to capture the pate areas as shown in Figure 3.

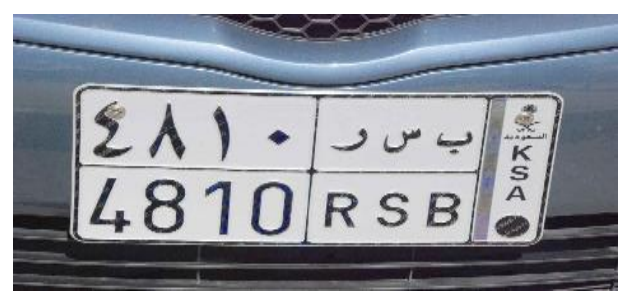

Fig.3. Acquiring the license plate area.

2- Extracting plate from car image.

In this step, the license plate is localized in the acquired image (see Figure 4) to process the recognition step on the license plates and removes unrelated areas.

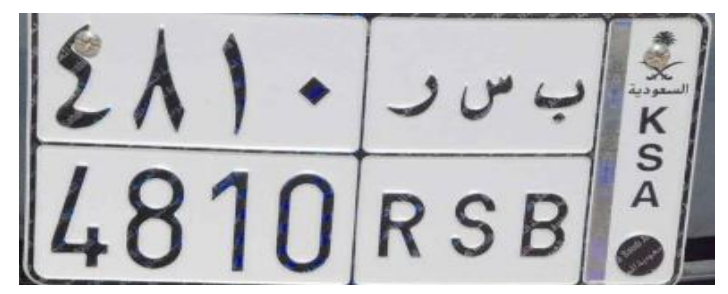

Fig.4. Localization of license plate.

3- Image segmentation, extract of characters from image.

Each character in the license plate is execrated in a separated image (see Figure 5). Then each character image is normalized, and some image processing algorithms could be applied that include convert image to black and white, and noise removal.

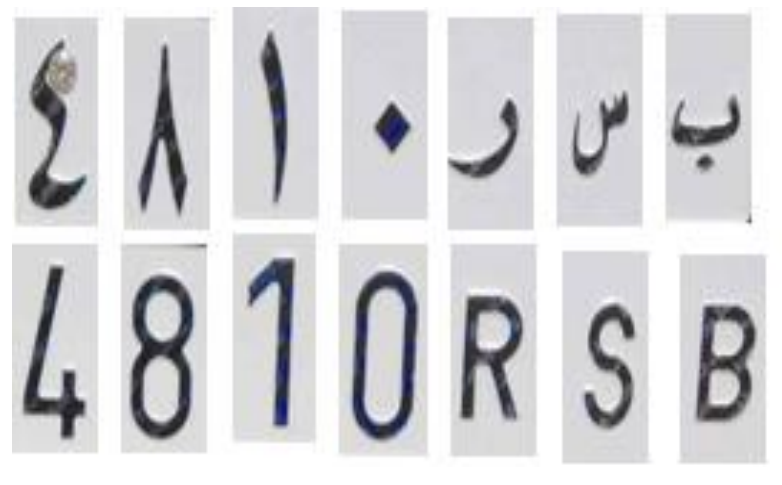

Fig.5. Segmentation of characters if license plates.

4- Character recognition.

Although Neural Network researchers are generally not concerned with whether their networks accurately resemble biological systems [21]. Neural network is the most common classifier that identify character images. A Neural Network is a processing device (algorithms or actual hardware) that is formed from processing units called neurons. A large Neural Network might have hundreds or thousands of processing units that are connected to form layers.

To achieve the objectives of the research the results of neural networks from Arabic and English character images will be integrated to improve the accuracy of LPR in Saudi Arabia. It should be noted that any other classifier could be used in the step including the deep learning approaches. 


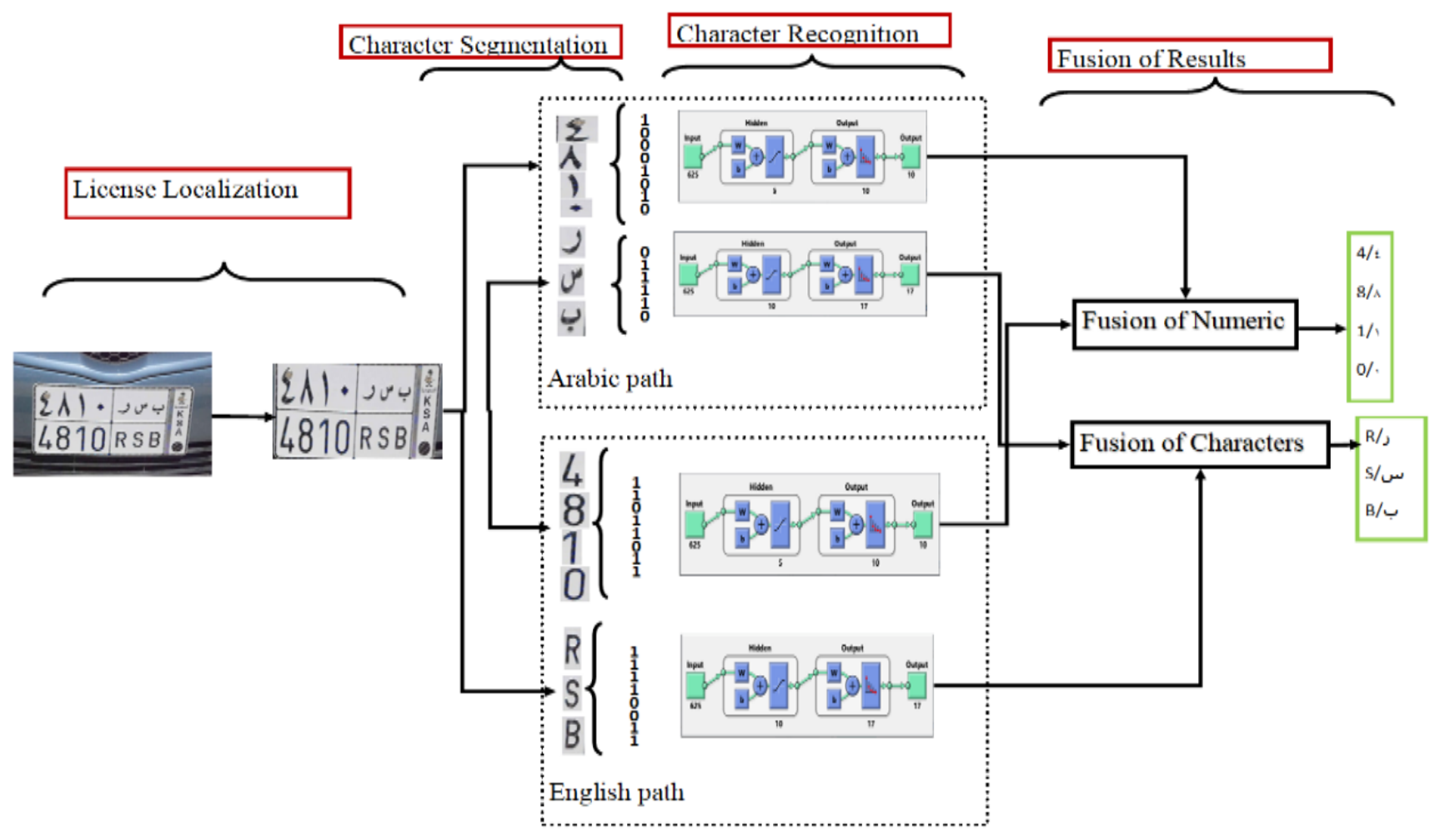

Fig.6. Graphical illustration of the fusion process for Saudi Arabia license plate

\section{Fusion of Saudi Arabia License Plate}

As described, Saudi Arabia license plate contains two paths of recognition, Arabic path and English path. Ideally both paths should give the same recognition results. However, the noise that could be added on the license plate through the recognition steps is text independent in which different recognition confidence of characters might be obtained as well as different recognition rates in each path. Moreover, identifying of characters in each path could be different. Therefore, both results can be combined to reach to the final recognition decision. The output of the neural network represents the confidence of the recognition to each character. We can simply multiply both results and then normalize the output to obtain the decision of the character images:

$\underbrace{\max }_{i} \frac{\prod o_{i A} o_{i E}}{\sum_{i}^{27} \Pi o_{i A} o_{i E}} i=0,1,2 \ldots, A, B, \ldots Z$

Where

$O_{i A}$ is the confidence of character $i$ in Arabic path. $O_{i E}$ is the confidence of character $i$ in English path.
The denominator of (1) represents a normalization term in which all character probabilities will sum up to 1 .

A block diagram of the proposed algorithms is show in Figure 6.

In Saudi Arabia license plate, numerical and alphabetical characters do not sit next to each other. Therefore, recognition of numerical characters could be obtained in a neural network, and recognition of alphabetical characters could be obtained in another neural network to reduce the number of classes at the output of the neural network. This could also improve the recognition rate. Therefore, we could have four neural networks, two for the Arabic path (numeric, alphabet) and two for the English path (numeric, alphabet). Equation (1) can be extended to

$$
\begin{aligned}
& \underbrace{\max }_{i} \frac{\Pi o_{i A} o_{i E}}{\sum_{i}^{17} \Pi o_{i A} o_{i E}} \quad i=A, B, \ldots Z \\
& \underbrace{\max }_{i} \frac{\Pi o_{i A} o_{i E}}{\sum_{i}^{10} \Pi o_{i A} o_{i E}} \quad i=0,1, \ldots, 9
\end{aligned}
$$



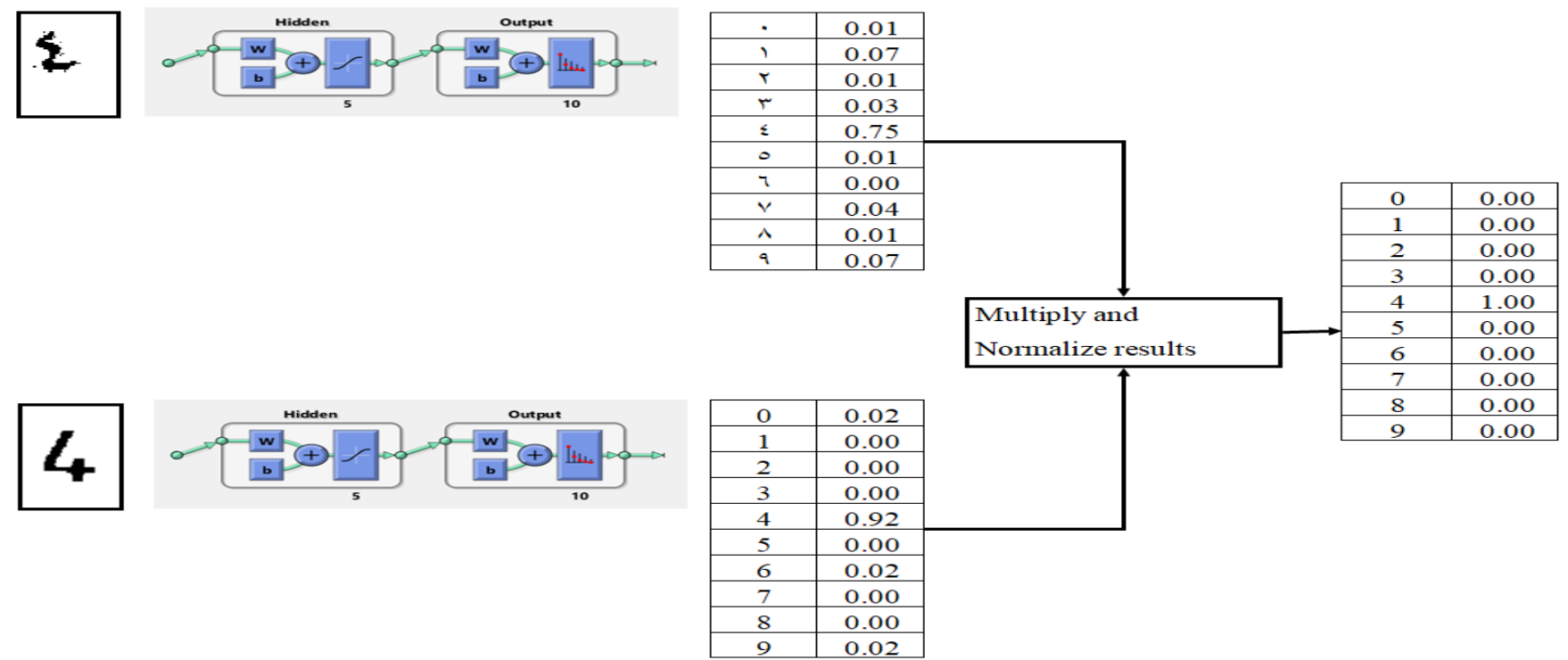

Fig.6. Graphical illustration of the fusion process for character $(4$, 乏)

Figure 6. shows a specific example of how the results from both neural networks are fused for the character image $(4, \varepsilon)$. The confidence from the Arabic path of Arabic image ( $\varepsilon$ ) is 0.75 and from the English path of English image (4) is 0.92 . Apparently, both paths can recognize the character correctly which is not necessarily true for all characters. However, the results of the fusion process increase the confidence of recognition to 1 . Because the confidence of the other digits is killed once they multiplied by their corresponding digits. The essence of the process is that the confidence of false alarm in both paths are independent, and these confidences will be eliminated if one path incorrectly recognized the character, leaving the true positive to have a quite significant value after the multiplication. The same process is applied for the alphabetical characters.

\section{Experimental Results}

The method has been tested on a local set because we are not able to find a public set of Saudi Arabia license plates. The data has been collected from different locations with different angles under different illumination conditions (daylight, shadow, night, etc.). We have collected 70 license plates that have been manually localized, also the characters have been manually segmented because the purpose of the method is to show how the two paths in Saudi Arabia license lates. The character images are then normalized to $(25 \times 25)$. The total number of obtained characters are shown in Table 2.

The parameters of the neural networks used in the experiments are as follows; the size of the input layer of all neural network would be 625 neurons. While the size of the output layer is set to 10 and 17 for numerical and character neural networks, respectively. We considered the function scaled conjugate gradient for training, and the nonlinear activation functions in the layers are tansig for first layer and softmax for the output layer.

Table 2. Local data set information.

\begin{tabular}{|l|l|l|l|l|}
\hline & \multicolumn{2}{|l|}{ Arabic } & \multicolumn{2}{l|}{ English } \\
\hline & Numeric & alphabetic & Numeric & alphabetic \\
\hline Train & 158 & 186 & 138 & 157 \\
\hline Test & 15 & 22 & 33 & 41 \\
\hline Total & 173 & 208 & 171 & 198 \\
\hline
\end{tabular}

The fusion process for numeric and alphabetic characters is achieved independently as one the characteristics of Saudi Arabia license plate that the separation between the numeric and alphabetic characters. Because we have different samples of Arabic and English image characters, we can synthesize the license plate by collecting data from the Arabic and English sets. The results were obtained for 1000 random combinations between Arabic and English sets. The number of nodes in 
the hidden layers of the neural networks were experimentally selected to 5 and 10 in the numeric and alphabetic neural networks, respectively. The error rates in recognition for the individual neural in Table. 3

Table 3. Error rate of recognition of individual neural network.

\begin{tabular}{|r|r|r|r|r|}
\hline \multicolumn{2}{|l|}{ Arabic } & \multicolumn{2}{l|}{ English } \\
\hline & numeric & alphabetic & numeric & alphabetic \\
\hline Train & 0.13 & 0.22 & 0.33 & 0.10 \\
\hline Test & 0.18 & 0.41 & 0.33 & 0.32 \\
\hline
\end{tabular}

fusion proses can improve the recognition using the The recognition error rates produced by the neural networks are quite significant, because the size of the training set is small, also the data itself contains error in which the shapes of the characters are distorted in some images. Adding the fusion mechanism will improve the performance of recognition in both numeric and alphabetic characters. Table 4 . shows the recognition error rates once including the fusion step to the output of the neural networks.

It is clearly shown that fusion will decrease the recognition error rate. Although the performance of the individual neural network is low, but the fusion process managed to improve the recognition in such way it takes the best from both paths. It should be noted that the fusion process improves the total performance regardless the classifier types, and performance of individual classifiers. Using neural network in the experiment is just an example of a classifier that could be used to show how the performance could be improved once adding the fusion mechanism.

\section{Conclusion}

The paper presented a fusion mechanism to combine the results of recognition of LPR system in Saudi Arabia license plates. The result of recognition is combined from the Arabic and English paths. Multiplying the outputs of neural networks can reduce the error produces in the individual neural network. The minimum improvement in the performance was $50 \%$ regarding to error rate. Moreover, the fusion process could be applied using other types of classifiers such as support vector machine, template matching, and Naïve Bayes. For future work, deep learning approach could be investigated in the recognition of Saudi Arabia license plate.

Table 4. Error rate of recognition using the fusion process.

\begin{tabular}{|c|c|c|c|c|c|}
\hline & \multirow{2}{*}{ 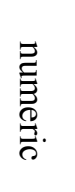 } & \multirow{2}{*}{$\begin{array}{l}\frac{\ddot{\sigma}}{\overrightarrow{0}} \\
\overrightarrow{0} \\
\stackrel{0}{0} \\
\overrightarrow{0} .\end{array}$} & \multirow{2}{*}{$\begin{array}{l}\underset{O}{\circ} \\
\stackrel{F}{F}\end{array}$} & \multicolumn{2}{|c|}{ Improvement \% } \\
\hline & & & & numeric & alphabetic \\
\hline Train & 0.09 & 0.11 & 0.10 & 72 & 50 \\
\hline Test & 0.10 & 0.26 & 0.18 & 70 & 56 \\
\hline
\end{tabular}

\section{References:}

[1] K. Aboura, R. Al-Hmouz, An overview of image analysis algorithms for license plate recognition, Organizacija, vol. 50, issue 3, pp. 285295.

[2] S. H. Park, K. I. Kim, K. Jung, H. J. Kim, Locating car license plates using neural network, IEE Electronics Letters, vol.35, no. 17, 1999, pp. 1475-1477.

[3] M. Yu and Y. D. Kim, 2000. An approach to Korean license plate recognition based on vertical edge matching, Systems, Man, and Cybernetics, IEEE International Conference, 2000, pp.2975 2980.

[4] K. K. Kim, J. B. Kim, K. I. Kim, H. J. Kim, Learning based approach for license plate recognition, Proceedings of IEEE Processing Society Workshop on Neural Networks for Signal Processing, vol. 2, 2000, pp: 614-623.

[5] C.N.E Anagnostopoulos, I. E Anagnostopoulos, V. Loumos, E. Kayafas, A License PlateRecognition Algorithm for Intelligent Transportation System Applications. IEEE Trans. Intelligent Transportation Systems, 7(3), 2006, pp. $377-392$.

[6] R. Al-Hmouz, K. Aboura, License plate localization using a statistical analysis of Discrete Fourier Transform signal, Computers \& Electrical Engineering, vol. 40, issue 3, pp. 982-992. 
[7] R. Al-Hmouz, S. Challa, License plate localization based on a probabilistic model, Machine Vision and Applications, vol. 21, issue 3, pp. 319-330.

[8] J. Guo and Y. Liu, "License Plate Localization and Character Segmentation With Feedback SelfLearning and Hybrid Binarization Techniques," in IEEE Transactions on Vehicular Technology, vol. 57, no. 3, 2008, pp. 1417-1424.

[9] J. Sharma, K. Saxena, and A. Sinhal, Comparative Study of Different Techniques for License Plate Recognition", Journal of Advanced Computing and Communication Technologies, vol. 1 no. 2, 2013, pp. 1-5.

[10] A. P. Nagare, License Plate Character Recognition System using Neural Network", International Journal of Computer Applications, vol. 25, no. 10, 2011, pp.36-39.

[11] M. Shidore, and S. P. Narote, 2011. Number Plate Recognition for Indian Vehicles, International Journal of Computer Science and Network Security, vol. 11, no. 2, 2011, pp. 143-146.

[12] R. Al-Hmouz, OCR Based Pixel Fusion, Journal of Applied Sciences, vol. 12, issue 22, 2012, pp. 2319-2325.

[13] M. Sarfraz and M. Jameel, License plate recognition system: Saudi Arabian case, in Computer-Aided Intelligent Recognition Techniques and Applications, M. Sarfraz, Ed. Chichester, UK: John Wiley \& Sons, ch. 2, 2005.

[14] Y. M. Alginahi, Automatic Arabic License Plate Recognition," International Journal of Computer and Electrical Engineering, vol. 3, no. 3, 2011, pp. 454-460, pp. 55-61.

[15] Z. Shaaban, An intelligent license plate recognition system, International Journal of Computer Science and Network Security, vol. 11, no.7, 2011. pp. 55-61.

[16] K. M. Almustafa, R. N. Zantout, and H. R. Obeid, Pixel density: Recognizing characters in Saudi license plates, in Proc. 10th International Conference on Intelligent Systems Design and Applications, 2010, pp. 308-313.

[17] K. M. Almustafa, R. N. Zantout, H. R. Obeid, and F. Sibai, Recognizing characters in Saudi license plates using character boundaries, in Proc. International Conference on Innovations in Information Technology, Abu Dhabi, 2011, pp. 415-420.

[18] K. Maglad, D. Mohamad, and N. A. Abulgasem, Saudian car license plate number detection and recognition using morphological operation and RBF neural network, Australian Journal of Basic and Applied Sciences, vol. 5, no. 12, 2011, pp. 1780-1786.

[19] K. W. Maglad, A vehicle license plate detection and recognition system, in Journal of Computer Science, vol.8, no. 3, 2012, pp. 310-315. [20] M. Deriche and M. Mohandes, A hybrid RFID-LPR system for vehicle access control during pilgrimage season in Saudi Arabia, in Proc. 9th International Multi-Conference on Systems, Signals and Devices, Chemnitz, 2018, pp. 1-6.

[21] Maureen Caudill "Neural Network Primer: Part I", AI Expert, Feb. 1989.

\section{Author Contributions:}

Hamdan Al-Harbi has implemented the Algorithm and paper organization.

Rami Al-Hmouz carried out the simulation.

\section{Creative Commons Attribution License 4.0 (Attribution 4.0 International, CC BY 4.0)}

This article is published under the terms of the Creative Commons Attribution License 4.0 https://creativecommons.org/licenses/by/4.0/deed.en US 